\title{
Modelagem constitutiva e caracterização empírica da interface revestimento asfáltico - base granular
}

\author{
Samuel de Almeida Torquato e Silva ${ }^{1}$, Jorge Barbosa Soares ${ }^{2}$, Suelly Helena de Araújo Barroso ${ }^{3}$ \\ ${ }_{1}^{1}$ Departamento de Engenharia de Transportes, Universidade Federal do Ceará (UFC), Fortaleza, Brasil, samuel@det.ufc.br \\ 2 Departamento de Engenharia de Transportes, Universidade Federal do Ceará (UFC), Fortaleza, Brasil, jsoares@det.ufc.br \\ ${ }^{3}$ Departamento de Engenharia de Transportes, Universidade Federal do Ceará (UFC), Fortaleza, Brasil, suelly@det.ufc.br
}

\section{Recebido:}

17 de abril de 2018

Aceito para publicação:

12 de julho de 2018

Publicado:

31 de agosto de 2018

Editor de área:

Francisco Thiago Aragão

Palavras-chaves: aderência, imprimação, interface

Keywords: adhesion, primer, interface

DOI:10.14295/transportes.v25i1.1661

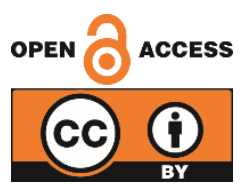

\section{RESUMO}

A relevância da interface revestimento asfáltico-base granular é bem estabelecida na literatura da Mecânica dos Pavimentos. Entretanto, há uma predominância da abordagem empírica no Brasil, o que não é compatível com métodos mecanísticos de dimensionamento de pavimentos. A fim de propor uma abordagem racional sobre o fenômeno de deslizamento entre as camadas em estudo, este artigo propõe um modelo constitutivo e um ensaio mecânico capaz de extrair os parâmetros de ruptura e rigidez deste modelo, utilizando o critério de Mohr-Coulomb e a Lei de Goodman, respectivamente. Os resultados experimentais corroboraram as premissas do modelo e o ensaio mostrouse de utilização viável para outros materiais. Finalmente, análises estruturais considerando o modelo desenvolvido foram realizadas utilizando o Método dos Elementos Finitos.

\begin{abstract}
The relevance of asphalt coat-granular base interface is well established in the Pavement Mechanics literature. However, there is a predominance of an empirical approach of characterization of this phenomenon in Brazil. Such approach is incompatible with mechanistic design methods. In order to propose a rational approach to deal with the slippage phenomenon within the asphalt coat-granular base layer interface, this paper suggests a constitutive model and a mechanical test able to assess strength and stiffness parameters of this model, by using Mohr-Coulomb failure criteria and Goodman's law, respectively. The experimental results support the model hypothesis and the test proved to be feasible to characterize other materials. Finally, structural analysis considering the model developed were performed through Finite Elements Method.
\end{abstract}

\section{INTRODUÇÃO}

A consideração sobre as condições de aderência entre camadas na pavimentacão fez-se necessária desde as primeiras formulações analíticas da Mecânica dos Pavimentos. Boussinesq (1885) solucionou o problema de um carregamento vertical, circular e estático sobre um meio semi-infinito e elástico linear, considerando apenas uma camada. Ao extrapolar a solução de Boussinesq para três camadas, Burmister (1943) teve que considerar como se daria a ligação entre as camadas, adicionando para isso uma premissa simplificadora: as camadas são perfeitamente aderidas e trabalham monoliticamente.

Quando se avalia a interface revestimento asfáltico-base granular, tal premissa equivale a afirmar que a imprimação constitui uma cola indeformável e irrompível, ou que o atrito entre estas camadas tende ao infinito. Tais premissas têm sido sistematicamente falseadas por 
ensaios de cisalhamento de interface. Mantilla e Button (1994) realizaram ensaios de cisalhamento direto e torção, dentre outros, a fim de mensurar a resistência da interface revestimento asfáltico-base granular, utilizando o modelo de ruptura de Mohr-Coulomb. Os autores, ao testarem diferentes ligantes de imprimação e condições de aplicação destes, obtiveram valores de coesão entre 0 e $300 \mathrm{kPa}$, e valores de 52 a $73^{\circ}$ para o ângulo de atrito. Esses resultados evidenciam que a ligação entre essas camadas apresenta uma relação de tensão e deformação. Goodman et al. (1968) estabeleceram em artigo sobre Mecânica das Rochas que a tensão de cisalhamento aplicada na interface e o deslocamento relativo entre camadas seguia uma relação linear (Equação 1):

$$
\tau=K_{t} \times \Delta u
$$

onde $\tau$ é o cisalhamento na interface, $\Delta u$ o deslocamento relativo entre as camadas e $K_{t}$ o módulo de reação transversal. A Figura 1 ilustra a Lei de Goodman aplicada ao deslizamento de uma camada asfáltica sobre uma base granular.

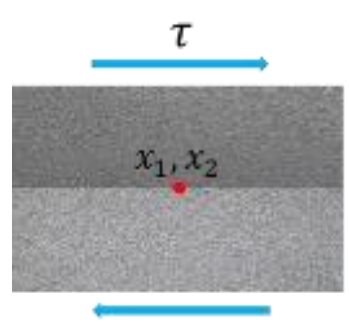

$\tau$
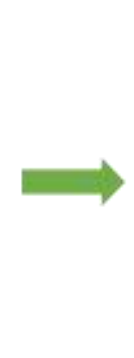

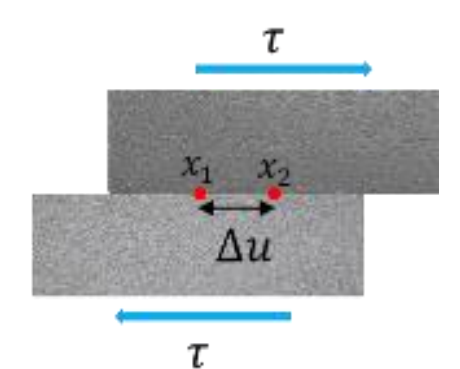

Figura 1. Lei de Goodman

A aderência é, portanto, mensurada através de $K_{t}$. No limite, se a hipótese de camadas perfeitamente aderidas fosse verdade, $K_{t}$ tenderia ao infinito. Diversos pesquisadores têm verificado o efeito de $K_{t}$ sobre as respostas estruturais do pavimento (Uzan et al., 1978; Khweir e Fordyce, 2003; Ziarie e Khabiri, 2007; Hu e Walubita, 2011; Scherer at al., 2017). É consenso que o valor adotado de $K_{t}$ possui considerável influência sobre as respostas estruturais do pavimento, como a magnitude das deformações de tração no revestimento, geralmente associadas ao trincamento por fadiga. Uzan et al. (1978) estabeleceram que para valores de $K_{t}$ menores que $10^{2} \mathrm{kPa} / \mathrm{mm}$, as camadas podem ser consideradas completamente descoladas, enquanto para valores maiores que $10^{5} \mathrm{kPa} / \mathrm{mm}$, as camadas podem ser consideradas perfeitamente aderidas.

Segundo Torquato e Silva et al.(2015), a aderência entre as duas camadas superiores do pavimento é muito mais relevante estruturalmente do que a aderência entre as camadas subjacentes. Possivelmente por esta razão, a literatura internacional costuma conter estudos sobre a aderência entre camadas asfálticas. Nos países desenvolvidos é comum a utilização de camadas duplas de mistura asfáltica. Entretanto, no Brasil, costumeiramente há apenas uma camada asfáltica assente sobre a base granular e os revestimentos são mais delgados, sendo também comum a adoção de tratamentos superficiais como primeiro revestimento. Os referidos autores demonstraram que a vida útil estimada do pavimento pode se elevar em até 3 vezes quando se passa de uma condição de camadas descoladas para aderidas.

As pesquisas nacionais acerca da imprimação, como Nogami et al. (1988) e Rabêlo (2006), costumam analisar a penetração do ligante de imprimação na base granular. Há, portanto, uma 
escassez de dados que descrevam mecanisticamente o comportamento da interface revestimento asfáltico-base granular. Mais recentemente, Almeida (2017) apresentou um trabalho mostrando a importância de se considerar também outros fatores, tais como: coesão, aderência e desgaste superficial.

O objetivo deste trabalho é propor um procedimento de caracterização e análise mecânica da interface revestimento asfáltico-base granular a fim de contribuir com o entendimento do fenômeno da aderência entre estas camadas a partir de uma análise mecanicista. Para o cumprimento deste objetivo, formulou-se um modelo constitutivo da interface dessas camadas e foi proposto um ensaio para extrair os parâmetros deste modelo. Em sequência, submeteu-se o procedimento aqui proposto para teste em materiais tipicamente utilizados em pavimentos no Estado do Ceará. Por fim, o modelo foi inserido em análise estrutural de pavimento com utilizando o Método dos Elementos Finitos.

\section{MATERIAIS UTILIZADOS}

\subsection{Base granular}

Utilizou-se uma base granular constituída de Brita Graduada Simples 3/4" (BGS), caracterizada por meio dos ensaios de granulometria (DNER ME 080/94), compactação (DNER-ME 162/94), CBR e expansão (DNER-ME 049/1994), bem como módulo de resiliência (DNIT 134/2016-ME), dentre outros, cujos resultados são apresentados na Tabela 1.

Tabela 1: Caracterização da BGS

\begin{tabular}{|c|c|c|c|c|c|c|c|c|}
\hline Material & $W_{o t}(\%)$ & $\begin{array}{l}\gamma_{S M a ́ x} \\
\left(\mathrm{~g} / \mathrm{cm}^{3}\right)\end{array}$ & $\begin{array}{l}\text { GC } \\
\text { (\%) }\end{array}$ & $\begin{array}{l}\text { MR } \\
\text { (MPa) }\end{array}$ & CBR(\%) & $\begin{array}{l}\text { Exp. } \\
\text { (\%) }\end{array}$ & $\begin{array}{l}\text { LL } \\
\text { (\%) }\end{array}$ & $\begin{array}{l}\text { LP } \\
\text { (\%) }\end{array}$ \\
\hline BGS & 7,8 & 2,156 & 100 & 478 & 64 & 0,01 & NL & NP \\
\hline
\end{tabular}

\subsection{Ligante de imprimação}

Para a imprimação utilizou-se asfalto diluído de petróleo de cura média (CM-30), cujas especificações foram fornecidas pela refinaria Petrobras/Lubnor. A taxa de aplicação utilizada foi de $1 \mathrm{~L} / \mathrm{m}^{2}$. A Tabela 2 exibe a caracterização do material.

Tabela 2: Caracterização do ligante de imprimação

\begin{tabular}{lll}
\hline Propriedades & Métodos & Resultados \\
\hline Viscosidade Cinemática a $60{ }^{\circ} \mathrm{C}$ (cSt) & D2170 & 55,4 \\
Ponto de fulgor $\left({ }^{\circ} \mathrm{C}\right.$ ) & $\mathrm{D} 3143$ & 41 \\
Destilado a $225^{\circ} \mathrm{C}(\%$ volume) & $\mathrm{D} 402$ & 19,5 \\
Destilado a $260^{\circ} \mathrm{C}(\%$ volume) & $\mathrm{D} 402$ & 50 \\
Destilado a $316^{\circ} \mathrm{C}$ (\% volume) & $\mathrm{D} 402$ & 82,9 \\
Resíduo a $360^{\circ} \mathrm{C}$ por diferença (\% volume) & $\mathrm{D} 402$ & 59 \\
Água por destilação (\% volume) & $\mathrm{D} 95$ & $<0,05$ \\
Solub. no Tricloroetileno no resíduo da destilação $(\%)$ & $\mathrm{D} 2042$ & 100 \\
Ductibilidade $15,5^{\circ} \mathrm{C}$ no resíduo da destilação $(\mathrm{cm})$ & $\mathrm{D} 113$ & 150 \\
\hline
\end{tabular}

\subsection{Mistura asfáltica}

A dosagem e a compactação da mistura asfáltica do tipo Concreto Asfáltico deram-se pela metodologia Marshall (DNER-ME 043/95). O ligante asfáltico CAP 50/70 foi proveniente da 
refinaria Petrobras/Lubnor e o tamanho máximo nominal (TMN) da mistura asfáltica é de $12,5 \mathrm{~mm}$.

\section{MODELAGEM CONSTITUTIVA DA INTERFACE}

Os ensaios de cisalhamento de interface como os realizados por Mantilla e Button (1994), normalmente detectam que a imprimação garante um ganho de aderência entre as camadas de revestimento e base. Porém, esta não é a única fonte de aderência. Segundo Lambe e Whitman (1995), as subcamadas granulares também apresentam aderência, sendo a diferença que neste caso a aderência é fornecida apenas pelo atrito entre os agregados.

Dessa forma, propõe-se neste artigo que a aderência entre revestimento asfáltico e base granular provenha de duas fontes, quais sejam: ligante de imprimação e atrito entre os agregados de ambas as camadas. Este segundo dependente da tensão compressiva sobre a interface. 0 módulo de reação transversal $K_{t}$, portanto, pode ser escrito da seguinte forma:

$$
\begin{gathered}
K_{t}=K_{i m p}+K_{f a t} \\
K_{f a t}=\alpha \sigma
\end{gathered}
$$

onde $K_{\text {imp }}$ representa a parcela da aderência fornecida pelo ligante de imprimação e $K_{f a t}$ a parcela fornecida pelo atrito entre os agregados das camadas, sendo este parâmetro descrito em função de uma constante $\alpha$ e da tensão de compressão $\sigma$. A Figura 2 representa graficamente as Equações 2 e 3.

A partir do mesmo raciocínio, o critério de ruptura de Mohr-Coulomb é adotado. A parcela de coesão desta envoltória é justificada pelo ligante de imprimação, enquanto a parcela dependente da tensão, pelo atrito entre os agregados das camadas, conforme descreve a Equação 4.

$$
\tau_{\max }=c+\sigma \tan \varphi
$$

Onde $\tau_{\text {max }}$ é a máxima tensão de cisalhamento, $c$ a coesão, $\sigma$ a tensão de compressão sobre a interface e $\varphi$ o ângulo de atrito. A Figura 3 mostra a envoltória de ruptura descrita pela Equação 4.

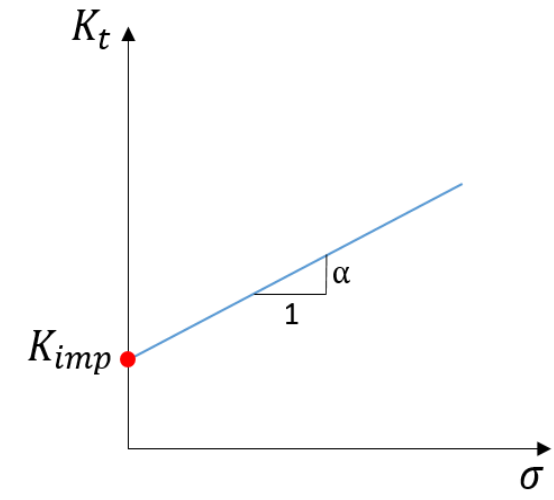

Figura 2. Módulo de reação transversal em função da tensão axial

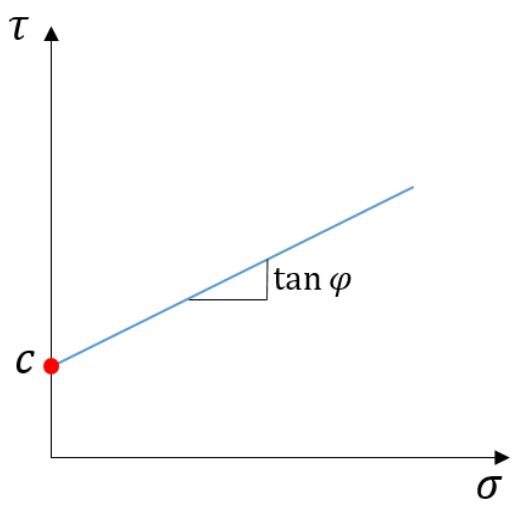

Figura 3: Envoltória de Mohr-Coulomb 
A modelagem da aderência, descrita pelo módulo de reação transversal $K_{t}$ e o critério de ruptura de Mohr-Coulomb, pode ser representada através de análogos mecânicos. A Figura descreve o modelo proposto de duas molas, uma para $K_{\text {imp }}$ e outra para $K_{f a t}$, e outro elemento para representar a ruptura da interface.

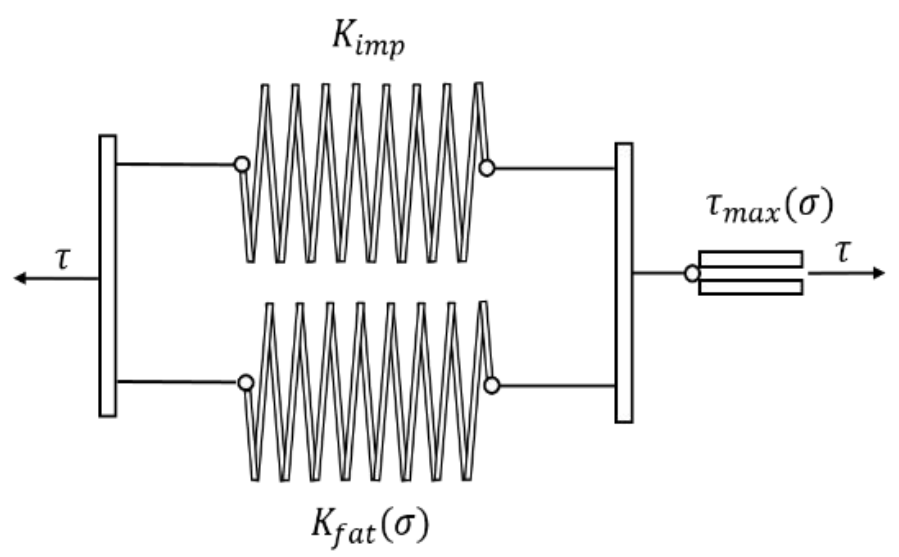

Figura 4: Modelo constitutivo proposto

\section{ENSAIO DE CISALHAMENTO DE INTERFACE}

$O$ procedimento do ensaio de cisalhamento desenvolvido na presente pesquisa se deu em duas etapas principais: a confecção do corpo de prova (CP) e o rompimento deste. Na primeira etapa, procurou-se reproduzir as condições de campo em termos de energia e umidade de compactação da camada de base, temperatura e taxa de aplicação do ligante, energia e temperatura de compactação da mistura asfáltica. Após moldado o CP, a ruptura da interface se deu pela aplicação monotônica da tensão de cisalhamento, estando a interface submetida aos diferentes níveis de tensão axial.

\subsection{Confecção do corpo de prova}

O molde construído para a confecção do CP foi constituído de dois cilindros vazados de $3 \mathrm{~cm}$ de altura e $5 \mathrm{~cm}$ de raio (Figura ). 0 cilindro inferior foi rosqueado à base metálica (Aço 1020), enquanto o cilindro superior se apoiou simplesmente sobre o inferior.

A primeira etapa da confecção do CP foi a compactação da base (NBR 7182/1986). Utilizouse a energia modificada correspondente a $2,7 \mathrm{MJ} / \mathrm{m}^{3}$. De posse da curva de compactação, o material de base foi compactado de modo que sua altura final fosse de aproximadamente $3 \mathrm{~cm}$ para que a interface ficasse exposta e pudesse ser rompida posteriormente. Eventuais imprecisões podem ser corrigidas, pois o rosqueamento do molde inferior torna sua altura ajustável.

Em seguida, pesou-se o $\mathrm{CP}$, para que fosse mantido em laboratório à temperatura ambiente até que perdesse $50 \%$ da umidade. Usualmente, este processo dura cerca de 1 dia, a depender do material e das condições ambientais.

A etapa seguinte foi a aplicação da imprimação. Para tal, deve-se varrer a superfície da amostra e irrigá-la com água à taxa de $1 \mathrm{~L} / \mathrm{m}^{2}$. Após 15 minutos de secagem, aplica-se o ligante de imprimação à taxa desejada. Para este artigo, aplicou-se $1 \mathrm{~L} / \mathrm{m}^{2}$ para todas as amostras. Por fim, espera-se $24 \mathrm{~h}$ para que o ligante de imprimação penetre na base. Em seguida, a camada asfáltica 
é aplicada (Figura ) e compactada sobre a base imprimada. Tal compactação foi realizada manualmente seguindo o método Marshall.

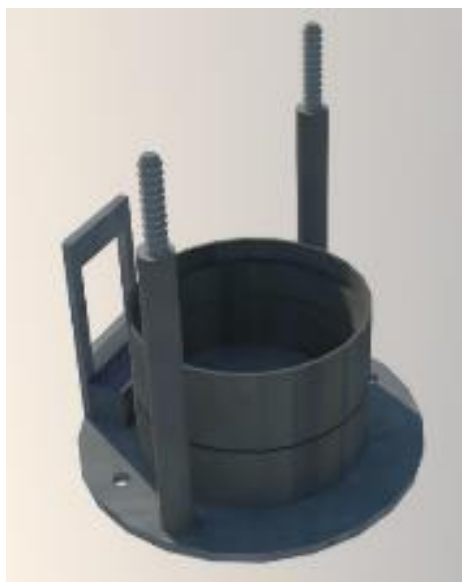

Figura 5: Molde de compactação

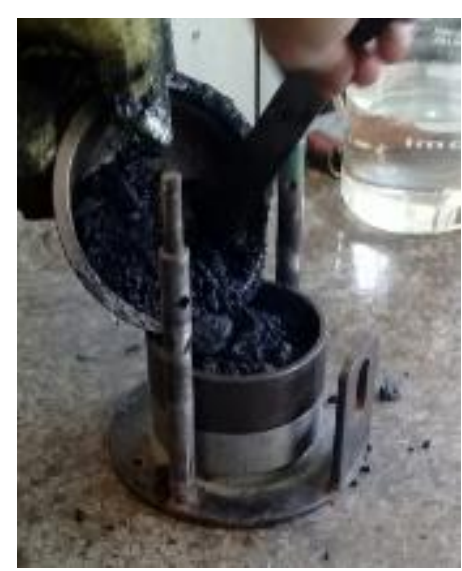

Figura 6: Aplicação da mistura asfáltica

\subsection{Aplicação do carregamento e ruptura do corpo de prova}

A tensão axial foi aplicada por meio de uma haste parafusada que transmite o esforço por meio de uma mola. De posse de sua constante elástica $(0,3 \mathrm{kN} / \mathrm{mm})$, a mola é utilizada como dinamômetro e pela medição de sua deformação infere-se a tensão axial aplicada na interface.

$$
\sigma=\frac{F}{A}=\frac{k \Delta x}{\pi r^{2}}
$$

Onde $\sigma$ é a tensão axial, $F$ a força axial, $A$ a área da seção transversal do $\mathrm{CP}, k$ a constante da mola, $\Delta x$ a deformação da mola e $r$ o raio da seção transversal do CP.

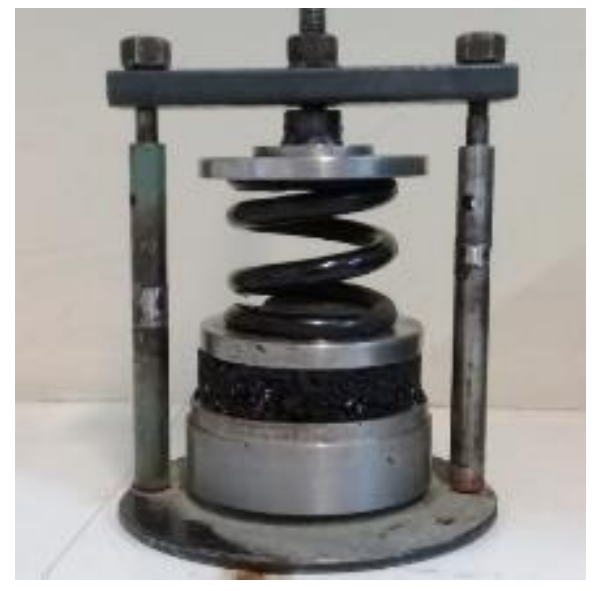

Figura 7: Aplicação do carregamento axial

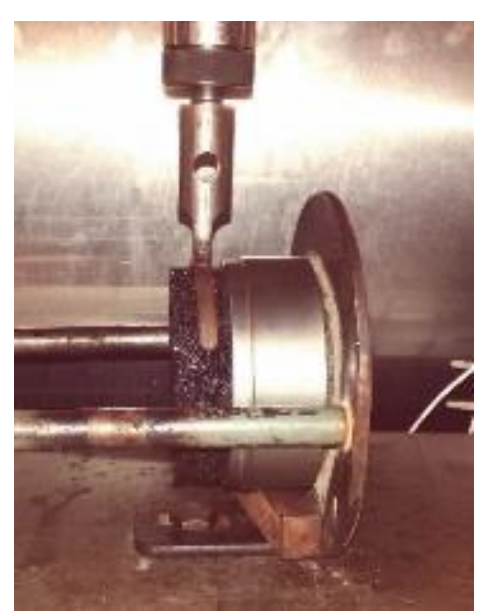

Figura 8: Aplicação da carga transversal

O carregamento transversal, responsável pelo cisalhamento da interface, foi aplicado pela Universal Testing Machine (UTM) por meio de uma garra a ela acoplada que se acomoda à força circular do CP. Esta carga foi aplicada de maneira monotônica à taxa de $20 \mathrm{kPa} / \mathrm{s}$. A garra deve ser posicionada de modo que fique próxima ao limite superior da interface, a fim de minimizar o braço de alavanca que vai da interface ao ponto de aplicação da carga. A Figura 8Erro! Fonte 
de referência não encontrada. ilustra a aplicação da carga transversal na condição de tensões axiais nulas.

Há, entretanto, que se considerar que parte das tensões de cisalhamento são absorvidas pelo atrito entre a placa de aço que recebe as tensões axiais e a mistura asfáltica. Através do uso de um plano inclinado aferiu-se o coeficiente de atrito dinâmico entre a placa e a mistura, sendo equivalente a 0,16 . Portanto, simplificadamente, para o cálculo da tensão de cisalhamento resistida pela interface, desconta-se $16 \%$ da tensão axial aplicada.

\section{EFEITO DA CONSIDERAÇÃO DO MODELO SOBRE A PREVISÃO DE ÁREA TRINCADA}

Uma vez que os parâmetros do modelo são conhecidos, é pertinente avaliar o efeito desses parâmetros sobre a vida útil do pavimento. Sendo estabelecido na literatura que a aderência precária entre camadas eleva as deformações de tração no revestimento (Ziari \& Khabiri, 2007, Torquato e Silva et al, 2015), torna-se razoável avaliar o efeito da consideração do modelo sobre a evolução de Área Trincada (AT) do revestimento, defeito comumente associado aos esforços de tração. Para esta avaliação, utiliza-se a função de transferência desenvolvida por Nascimento (2015) a partir de três condições de aderência, quais sejam: aderência perfeita $\left(K_{t} \rightarrow \infty\right)$, uma condição intermediária representada pelo modelo e aderência nula $\left(K_{t} \approx 0\right)$.

$$
\begin{aligned}
A T(\%) & =A \times D_{\text {red }}{ }^{B} \\
D_{\text {red }} & =D \times S \\
D & =\frac{N}{N_{f}}
\end{aligned}
$$

Onde A e B são coeficientes de regressão, iguais a 7272,68 e 8,663, respectivamente. Dred é o dano reduzido, $\mathrm{D}$ é o dano médio e $\mathrm{S}$ é uma função de ajuste do método. 0 dano $\mathrm{D}$ é a razão entre o número de repetições de cargas $\mathrm{N}$ e o número total de cargas $\mathrm{N}_{\mathrm{f}}$ que o material suporta, sendo este número função da deformação principal de tração e dos parâmetros de vida de fadiga Y (20749123), $\Delta(-1,43), \beta(-0,30), \alpha(3,23), C 11(0,000626)$ e C12 (0,617111). Os parâmetros são extraídos do ensaio de fadiga por tração direta (Nascimento, 2015).

A estrutura analisada (Tabela 3) é submetida a $1,93 \times 10^{7}$ repetições de eixo padrão (raio de $10,8 \mathrm{~cm}$ e carga de $550 \mathrm{kPa}$ ) ao longo de 120 meses. Considera-se que a estrutura é axissimétrica e todas as camadas são elásticas lineares, com exceção da interface, que segue o modelo proposto neste artigo. Devido à não linearidade provocada pela interface, utiliza-se o Método dos Elementos Finitos. Maiores detalhes sobre o método de análise podem ser encontrados em Torquato e Silva (2017), inclusive a modelagem do elemento finito de interface.

Tabela 3: Estrutura analisada

\begin{tabular}{llll}
\hline Camadas & Espessuras & E (MPa) & Poisson \\
\cline { 1 - 3 } Revestimento & 5 & 3000 & 0,30 \\
Interface & -------- & ------- & ------- \\
Base & 15 & 478 & 0,35 \\
Subbase & 15 & 493 & 0,40 \\
Subleito & $\infty$ & 407 & 0,40 \\
\cline { 1 - 2 }
\end{tabular}

\section{DISCUSSÃO DOS RESULTADOS}

\subsection{Caracterização da interface}

A Figura 9 demonstra a relação existente entre o cisalhamento máximo suportado pela interface e a tensão axial sobre ela aplicada (compressão positiva). A relação crescente suporta a 
hipótese de que a envoltória de Mohr-Coulomb descreve a resistência ao cisalhamento desta interface, bem como a pequena variação dos pontos sujeitos ao mesmo nível de tensão axial sugere a reprodutibilidade do ensaio. Tanto os valores de coesão $(45,77 \mathrm{kPa})$ quanto de ângulo de atrito $\left(62,9^{\circ}\right)$ estão dentro dos limites dos resultados obtidos por Mantilla e Button (1994).

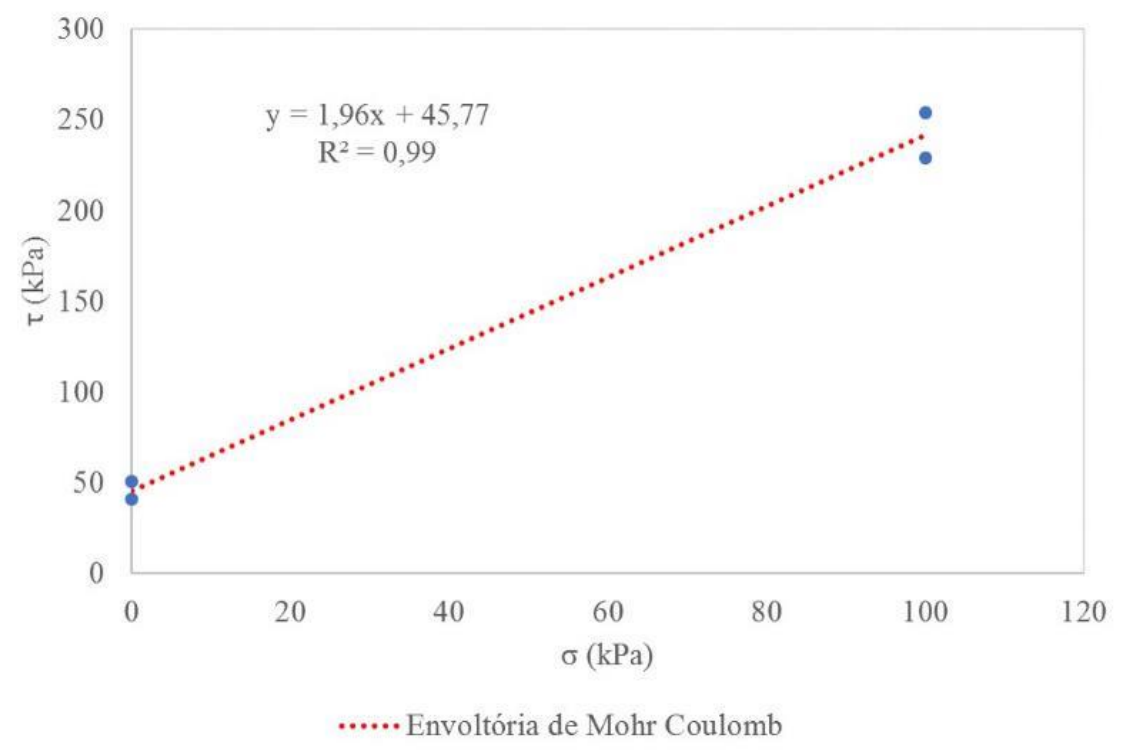

Figura 9: Envoltória de ruptura da interface

No que diz respeito ao módulo de reação transversal $\left(K_{t}\right)$, o gráfico apresentado na Figura 10 corrobora a hipótese de que esta variável é função da tensão axial aplicada sobre a interface, mas que há também uma parcela independente, quando a tensão axial é nula. Estes resultados concordam com o modelo proposto, uma vez que a parcela de $K_{t}$ dependente da tensão axial, representada por $K_{\text {fat }}$ (Equação 2), é modelada pela Equação 3, enquanto o termo independente é representado por $K_{i m p}$, correspondente à contribuição do ligante de imprimação no ganho de aderência. A Tabela4 apresenta a caracterização completa da interface, conforme os 4 parâmetros $\left(K_{i m p}, \alpha, C, \varphi\right)$ do modelo proposto.

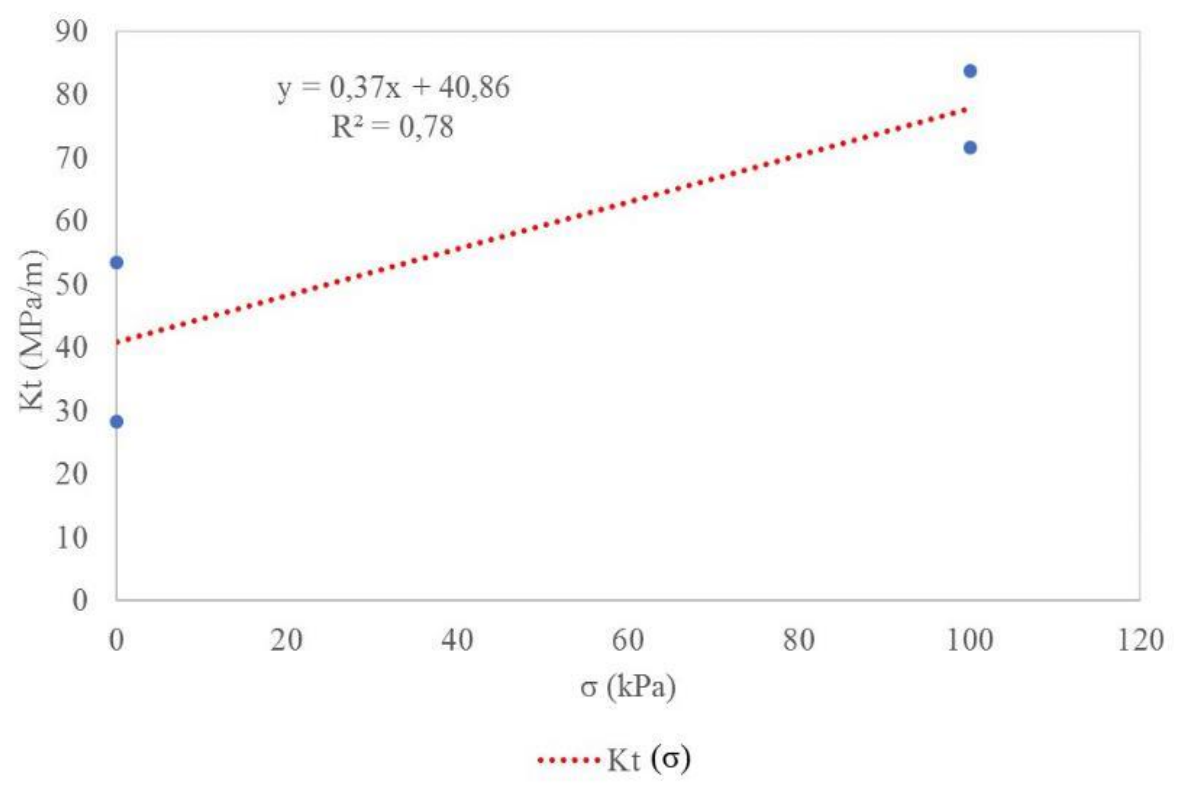

Figura 10: Curva do módulo de reação transversal 
Tabela 4: Caracterização da interface

\begin{tabular}{ccccccc}
\hline $\begin{array}{c}\sigma \\
(\mathbf{k P a})\end{array}$ & $\begin{array}{c}\mathbf{K}_{\mathbf{t}} \\
(\mathbf{M p a} / \mathbf{m})\end{array}$ & $\begin{array}{c}\boldsymbol{\tau} \\
(\mathbf{k P a})\end{array}$ & $\begin{array}{c}\boldsymbol{K}_{\boldsymbol{i m p}} \\
(\mathbf{k P a} / \mathbf{m m})\end{array}$ & $\begin{array}{c}\boldsymbol{\alpha} \\
\left(\mathbf{m m}^{-\mathbf{1}}\right)\end{array}$ & $\begin{array}{c}\mathbf{C} \\
(\mathbf{k P a})\end{array}$ & $\begin{array}{c}\boldsymbol{\Phi} \\
\left.\mathbf{(}^{\circ}\right)\end{array}$ \\
\hline 0 & $0,28 \times 10^{2}$ & 41,00 & & & & \\
0 & $0,54 \times 10^{2}$ & 50,55 & & & & \\
100 & $0,72 \times 10^{2}$ & 228,67 & 40,86 & 0,37 & 45,77 & 62,93 \\
100 & $0,84 \times 10^{2}$ & 254,14 & & & & \\
\hline
\end{tabular}

É interessante confrontar os resultados com os limites propostos por Uzan et al. (1978) para qualificar a interface ensaiada quanto à sua importância estrutural. Segundo os autores, as camadas podem ser consideradas aderidas se $K_{t} \geq 10^{5} \mathrm{kPa} / \mathrm{mm}$, por outro lado, se $K_{t} \leq$ $10^{2} \mathrm{kPa} / \mathrm{mm}$, podem ser consideradas descoladas. Conforme mostra a Figura 11, a interface ensaiada se aproxima mais da condição descolada, o que significa que ela teria reduzida relevância estrutural num pavimento. Entretanto, deve-se ressaltar que esta conclusão é apenas indicativa e se limita aos materiais ensaiados e ao tipo de revestimento estudado. Adicionalmente, a influência da aderência na interface sobre a resposta estrutural varia conforme os materiais utilizados na estrutura, espessuras adotadas e outros fatores (Torquato e Silva et al., 2015).

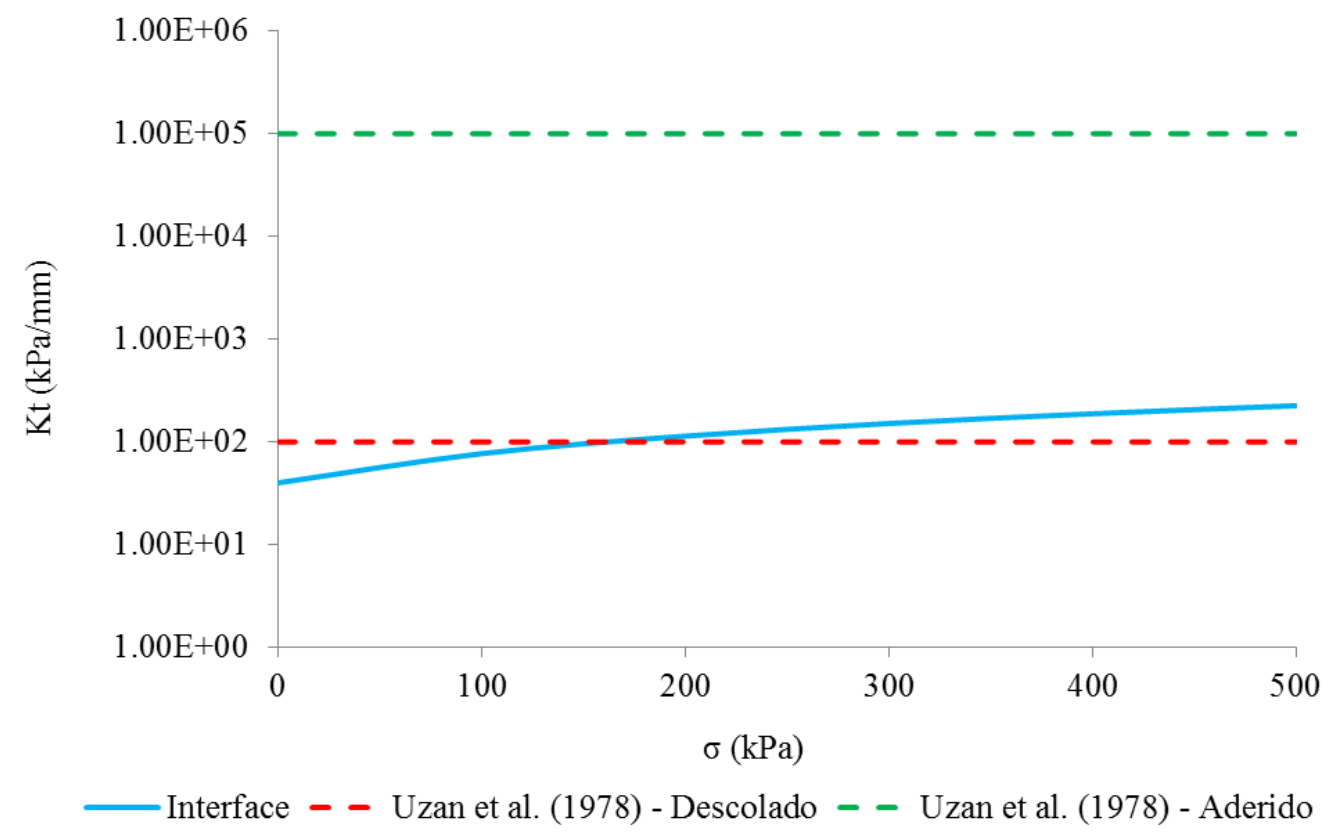

Figura 11: Comparação entre a curva de $K_{t}$ da interface e os limites de Uzan et al. (1978)

\subsection{Previsão de Área Trincada}

De posse dos parâmetros do modelo, é possível utilizá-los como dados de entrada na análise. Na Figura 12 observa-se que a curva do modelo tem mais proximidade com a curva da condição de aderência nula, o que é coerente com os limites de Uzan et al. (1978) expostos na Figura 12. 


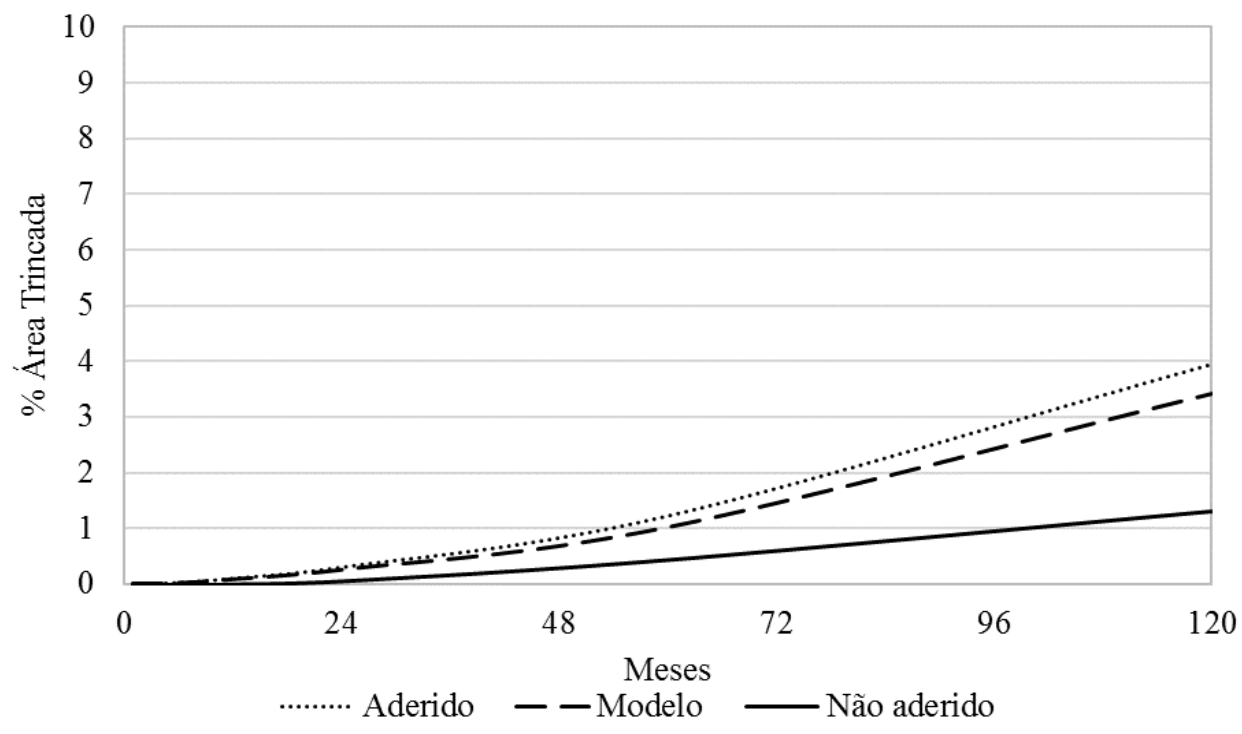

Figura 12: Evolução de área trincada para diferentes condições de aderência

Para efeitos práticos e de simplificação, seria mais realista considerar que as camadas ligadas pela interface em estudo estariam descoladas. Entretanto, deve-se fazer a ressalva de que este resultado não pode ser extrapolado para todas as interfaces entre revestimento asfáltico e base granular, sendo correspondentes apenas às condições de análise e experimentais deste artigo. Isto é, diferentes combinações de revestimento, imprimação e base granular devem apresentar desempenho estrutural diferente do apresentado na Figura 12.

\section{CONCLUSÕES}

O ensaio proposto cumpriu o objetivo de caracterizar a interface reproduzindo as condições de aplicação dos materiais em campo no que se refere à temperatura do revestimento asfáltico, energia de compactação e umidade do material granular. Além disso, o processo desde a confecção até a ruptura dos corpos de prova durou 1 semana, o que tornou o ensaio prático para ser utilizado em pesquisa, inclusive para futuro teste e desenvolvimento de novos materiais de imprimação.

No que diz respeito ao modelo constitutivo, pode-se concluir que os resultados empíricos deram suporte à formulação proposta, assim como dependência dos parâmetros de resistência e módulo de reação transversal em relação à tensão axial foi confirmada. Tal modelo é uma importante contribuição deste trabalho, pois seus parâmetros podem ser utilizados em simulação computacional, o que viabiliza a consideração da interface de maneira mais realista em métodos mecanístico-empíricos de dimensionamento.

A interface estudada mostrou-se ineficaz estruturalmente, para a solução de base BGS e revestimento do tipo CA testado. Porém, isso não deve ser um fator que desestimule a pesquisa acerca da aderência entre outros tipos de camadas constituídas de diferentes materiais. Pelo contrário, reforça a importância do estudo de outros materiais existentes e desenvolvimentos de novos, pois a modelagem apropriada da interface confere maior precisão ao dimensionamento do pavimento, e o desenvolvimento de novos materiais pode aproximar a interface à condição de aderência perfeita. Investigações com revestimentos mais esbeltos, como os tratamentos superficiais devem ser incentivadas, tendo em vista a função estrutural destes ser diferenciada em análise mecanística-empírica.

0 resultado da previsão de área trincada corroborou as conclusões parciais do ensaio que, baseadas nos limites de Uzan (1978), afirmavam que a interface em estudo apresentaria 
desempenho mais próximo da condição de camadas não aderidas. Apesar dos resultados promissores, análises considerando configurações estruturais, tais quais diferentes espessuras e materiais, bem como cargas transversais são recomendados para melhor entendimento do fenômeno.

Conclui-se que o método de caracterização proposto apresentou resultados promissores para a modelagem da interface revestimento asfáltico-base granular. Entretanto, é necessária a realização de ensaios com uma maior diversidade de materiais a fim de garantir a reprodutibilidade e a aplicabilidade do procedimento aqui apresentado quando sujeito a diferentes condições.

\section{REFERÊNCIAS}

Boussinesq, J. (1885). Application des pontentiels à l'étude de l'Equilibre et du Movement des Solides Elastiques.

Burmister, D. (1943). The theory of stresses and displacement systems and applications to the design of airport runways.

Goodman, R., Taylor, R., \& Brekke, T. (1968). A model for the mechanics jointed rock. Transportation Research Board, 637-659.

Hu, X., \& Walabita, L. (2011). Effects of layer interfacial bonding conditions on the mechanistic responses in asphalt pavements. Journal of Transportation Enginnering, 28-36. DOI: 10.1061/(ASCE)TE.1943-5436.0000184

Khweir, K., \& Fordyce, D. (2003). Influence of layer bonding on the prediction of pavement. Proceedings of the Institution of Civil Engineers: Transport, 73 - 83. DOI: 10.1680/tran.2003.156.2.73

Lambe, T., \& Whitman, R. (1995). Soil Mechanics 2.ed. Nova York: John Wiley \& Sons Inc.

Mantilla, C., \& Button, J. (1994). Prime coat methods and materials to replace cutback asphalt. FHWA - Research Report 1334 1F. Austin, Texas.

Nascimento, L. (2015). Implementation and Validation of the Viscoelastic 8 Continuum Damage Theory for Asphalt Mixture and Pavement Analysis in Brazil. Tese de Doutorado. Carolina do Norte, EUA.

Nogami, J., Villibor, D., \& Fabbri, G. (1988). Imprimadura asfáltica em bases de solo arenoso fino laterítico. Anais da XV Reunião Anual de Pavimentação. Florianópolis.

Rabêlo, A. (2006). Contribuição ao estudo da imprimação betuminosa das bases rodoviárias do estado do Ceará. Dissertação de Mestrado. Fortaleza, Ceará, Brasil: Universidade Federal do Ceará.

Scherer, R., Bueno, L., Pereira, D., Specht, L., \& Trindade, G. (2017). Influência da aderência entre camadas no desempenho esperado de pavimentos asfálticos. Anais da XXX ANPET. Rio de Janeiro.

Torquato e Silva, S. (2017). Modelagem mecanístico-empírica da interface revestimento asfáltico-base granular. Dissertação de Mestrado. Fortaleza, Ceará, Brasil: Universidade Federal do Ceará.

Torquato e Silva, S., J. Bastos, \& Soares, J. (2015). Influência da aderência na análise de pavimentos asfálticos. 44ํㅡAPv - 18oㅜ ENACOR. Foz do Iguaçu, Brasil.

Uzan, J., Livneh, M., \& Eshed, Y. (1978). Investigation of adhesion properties between asphaltic concrete layers. Transportation Research Board, 495-521.

Ziari, H., \& Khabiri, M. (2007). Interface condition influence on prediction of flexible pavement life. Journal of Civil Engineering and Management, 71-76. DOI: 10.1080/13923730.2007.9636421 